\title{
Short communication: Genetic variation in choice consistency for cows accessing automatic milking units
}

\author{
Peter Løvendahl, ${ }^{* 1}$ Lars Peter Sørensen, $†$ Martin Bjerring, $\ddagger$ and Jan Lassen $†$ \\ ${ }^{*}$ Centre for Quantitative Genetics and Genomics, Department of Molecular Biology and Genetics, Aarhus University, DK 8830 Tjele, Denmark \\ †Nordic Cattle Genetic Evaluation, SEGES, DK-8200 Aarhus N, Denmark \\ łDepartment of Animal Science, Aarhus University, DK 8830 Tjele, Denmark
}

\section{ABSTRACT}

Dairy cows milked in automatic milking systems (AMS) with more than 1 milking box may, as individuals, have a preference for specific milking boxes if allowed free choice. Estimates of quantitative genetic variation in behavioral traits of farmed animals have previously been reported, with estimates of heritability ranging widely. However, for the consistency of choice in dairy cows, almost no published estimates of heritability exist. The hypothesis for this study was that choice consistency is partly under additive genetic control and partly controlled by permanent environmental (animal) effects. The aims of this study were to obtain estimates of genetic and phenotypic parameters for choice consistency in dairy cows milked in AMS herds. Data were obtained from 5 commercial Danish herds (I-V) with 2 AMS milking boxes (A, B). Milking data were only from milkings where both the present and the previous milkings were coded as completed. This filter was used to fulfill a criterion of free-choice situation (713,772 milkings, 1,231 cows). The lactation was divided into 20 segments covering $15 \mathrm{~d}$ each, from 5 to $305 \mathrm{~d}$ in milk. Choice consistency scores were obtained as the fraction of milkings without change of box [i.e., $1.0-\mu$ (box change)] for each segment. Data were analyzed for one part of lactation at a time using a linear mixed model for first-parity cows alone and for all parities jointly. Choice consistency was found to be only weakly heritable (heritability $=0.02$ to 0.14 ) in first as well as in later parities, and having intermediate repeatability (repeatability coefficients $=0.27$ to 0.56 ). Heritability was especially low at early and late lactation states. These results indicate that consistency, which is itself an indication of repeated similar choices, is also repeatable as a trait observed over longer time periods. However, the genetic background seems to play

\footnotetext{
Received April 8, 2016.

Accepted August 18, 2016.

${ }^{1}$ Corresponding author: Peter.Lovendahl@mbg.au.dk
}

a smaller role compared with that of the permanent animal effects, indicating that consistency could also be a learned behavior. We concluded that consistency in choices are quantifiable, but only under weak genetic control.

Key words: choice consistency, automatic milking system milking, heritability

\section{Short Communication}

Dairy cows milked in automatic milking systems (AMS) with more than 1 milking box may, as individuals, have a preference for specific milking boxes if allowed free choice. Previously, it has been reported that cows have consistent preferences for right or left sides of milking parlors (Paranhos da Costa and Broom, 2001; Grasso et al., 2007), and that parlor side preferences persist over several months (Hopster et al., 1998). Hopster et al. (1998) also found that cows with strong side preferences show various stress responses, such as reduced yield, when milked in the nonpreferred side of the parlor. Similarly, in herds using AMS, for individual cows with strong milking box preferences the milking process could be affected if milked in the nonpreferred milking box. However, reports on milking box preferences and consistency in time are scarce, and because AMS use is becoming increasingly common this deserves further study.

The choice between 2 available milking boxes represents a choice situation where, ideally, the 2 alternatives are equally attractive to the cow. Several external factors may affect the choice in the given situation, such as previous experience and training as well as construction detail differences that are not obvious to humans. Individual animals may vary between the 2 extremes of complete consistency and changing boxes at every milking, thereby adopting a favorite box or flexible box choice patterns. Similar choice categories and intermediates were described by Hopster et al. (1998) with a continuous range of values assigned as consistency scores. By assuming that consistency is an attribute of 
Table 1. Overview of data for first parity and multiparous cows, overall and within 4 stages of lactation (DIM section; means \pm SD)

\begin{tabular}{lcccccc}
\hline Item & $\begin{array}{c}\text { Cows, } \\
\text { no. }\end{array}$ & $\begin{array}{c}\text { Records, } \\
\text { no. }\end{array}$ & $\begin{array}{c}\text { Choice consistency, } \\
\text { score }\end{array}$ & $\begin{array}{c}\text { Milk yield, } \\
\mathrm{kg} / \mathrm{d}\end{array}$ & $\begin{array}{c}\text { Box time, } \\
\mathrm{min} / \mathrm{d}\end{array}$ & $\begin{array}{c}\text { Milking frequency, } \\
\text { no. } / 24 \mathrm{~h}\end{array}$ \\
\hline Primiparous & & & & & & \\
5-50 DIM & 493 & 1,384 & $0.67 \pm 0.15$ & $29.3 \pm 5.1$ & $20.3 \pm 5.6$ & $2.76 \pm 0.57$ \\
51-110 DIM & 470 & 1,694 & $0.64 \pm 0.15$ & $32.2 \pm 4.6$ & $21.4 \pm 5.2$ & $2.76 \pm 0.57$ \\
111-215 DIM & 530 & 2,970 & $0.62 \pm 0.16$ & $30.3 \pm 4.6$ & $18.2 \pm 4.6$ & $2.70 \pm 0.55$ \\
216-305 DIM & 470 & 2,307 & $0.60 \pm 0.15$ & $28.0 \pm 4.6$ & $16.2 \pm 3.7$ & $2.53 \pm 0.48$ \\
All & 730 & 8,355 & $0.63 \pm 0.16$ & $29.9 \pm 4.9$ & $18.6 \pm 5.1$ & $2.73 \pm 0.57$ \\
Multiparous & & & & & & \\
5-50 DIM & 873 & 2,449 & $0.66 \pm 0.16$ & $34.1 \pm 8.0$ & $21.0 \pm 5.8$ & $2.93 \pm 0.65$ \\
51-110 DIM & 883 & 3,225 & $0.63 \pm 0.16$ & $36.5 \pm 7.0$ & $21.6 \pm 5.5$ & $3.02 \pm 0.64$ \\
111-215 DIM & 990 & 5,756 & $0.61 \pm 0.16$ & $33.3 \pm 6.1$ & $18.9 \pm 4.9$ & $2.75 \pm 0.57$ \\
216-305 DIM & 854 & 4,247 & $0.61 \pm 0.16$ & $29.4 \pm 5.6$ & $16.8 \pm 4.1$ & $2.53 \pm 0.50$ \\
All & 1,231 & 15,677 & $0.62 \pm 0.16$ & $33.0 \pm 7.0$ & $19.2 \pm 5.3$ & $2.76 \pm 0.61$ \\
\hline
\end{tabular}

the animal, and that individuals differ in this aspect, consistency may be partly under genetic control and partly controlled by permanent environmental (animal) effects, including learned behavior (e.g., Rioja-Lang et al., 2012). Estimates of quantitative genetic variation in behavioral traits of farmed animals have previously been reported with estimates of heritability ranging widely (e.g., aggression and maternal behavior in sows, Løvendahl et al., 2005; time budgets in dairy cows, Løvendahl and Munksgaard, 2016). However, for choice consistency, we found no published estimates of heritability for dairy cows.

Individual and genetic variation has been shown for several traits related to the milking process, such as yield per milking and per day, milking frequency, flow rates, and time used in the milking box (e.g., Løvendahl and Chagunda, 2011; Løvendahl et al., 2012). These traits are correlated at the genetic and phenotypic level, but their relationship with choice consistency has so far only been briefly reported (Hopster et al., 1998; Paranhos da Costa and Broom, 2001), and not for AMS choice situations. Our hypothesis was that choice consistency is partly under additive genetic control and partly controlled by permanent environmental (animal) effects, and that consistency at all levels is correlated with a range of other milking traits. The aims of our study were to estimate genetic and phenotypic parameters for choice consistency in dairy cows milked in AMS herds and for correlated milking traits of the same cows. To do so, a cohort study was undertaken using detailed milking data obtained from 5 Danish herds, each keeping cows in a single group with free access to 2 milking boxes.

Data were obtained from 5 commercial Danish herds (I-V) with 2 AMS milking boxes (A, B; DeLaval VMS, DeLaval, Tumba, Sweden) and keeping Holstein cows of mixed parities in single groups. There was no general training scheme for cows before their first calving. The cows were trained to AMS when newly calved in first parity during 2 daily sessions over a few days where they were fetched and guided into any available box, thus usually being introduced to both boxes. There were 730 cows in first parity, and 1,231 cows when considering parities 1 to 4 (Table 1 ). The data were retrieved over a period of $1 \mathrm{yr}$ in herd I and $2 \mathrm{yr}$ in herds II to V. Data were downloaded from the herd-management software (Delpro, DeLaval) and only records coded as milkings were used. The data contained information about box identification, animal identification, date and time for each milking, and total milk yield. The number of milkings per day per milking box varied between herds from a mean of 86 to 132 with a standard deviation of 11 to 21 . Within herd the 2 boxes were visited equally and the number of daily milkings varied little with season. Parity number and stage of lactation, as DIM, was obtained as supplementary information by linking to the national cattle database, which was also used for tracing ancestries for genetic analysis, giving a total of 16,089 animals in the pedigree (Nordic Cattle Genetic Evaluation, SEGES, Skejby, Denmark).

Variables were derived from each milking after filtering on a completion code from the management software splitting milkings into complete and incomplete groups. Following an incomplete milking, the cow will have immediate access to be milked again; thus, overriding system settings for minimum required milking intervals. Consequently, the subsequent milking of the same cow after an incomplete milking was assigned to a category of disturbed milkings, using the approach of Løvendahl and Chagunda (2011). Only data from undisturbed milkings were then used for the statistical analysis to fulfill a criterion of free-choice situation.

At each milking the milking interval in hours was calculated and also expressed as milking frequency per $24 \mathrm{~h}$ (MF). Milk yield was measured per milking (MY). Milk yield per day (MY24) was then calculated using a curve-linear extrapolation formula (Løvendahl and Chagunda, 2011): 


$$
\begin{aligned}
\mathrm{MY} 24 & =-0.1745+0.6213 \times \mathrm{MF}+\mathrm{MY} \\
& \times(0.2806+0.8118 \times \mathrm{MF}),
\end{aligned}
$$

where MY24 is the calculated milk yield per $24 \mathrm{~h}$, MF is the milking frequency for the current milking, and MY is the yield recorded at the current milking. At each milking, the total time spent in the milking box was calculated, and similarly the total box time per day was calculated by extrapolation (i.e., multiplication by MF).

The lactation was divided into 20 segments covering $15 \mathrm{~d}$ each, from 5 to 305 DIM (Løvendahl and Chagunda, 2011). Milking traits were then expressed per segment by taking mean values as input for statistical analysis. Segments having less than 6 records were omitted from further analysis. The mean milking date for each segment was used to assign test-day effects to segment mean values in the following analysis. These were further condensed into 1-mo herd-year-seasons. The lactation was also divided into 4 larger sections to investigate how traits changed through the lactation. These sections were named start (DIM 5-50; segments 1-3), early (51-110; segments 4-7), mid (111-215; segments 8-14), and late (216-305; segments 15-20).

The choice consistency scores (CCS) were obtained as the fraction of milkings without change of box since last milking [i.e., CCS $=1.0$ - (number of box changes/ total number of milkings)] for each segment, similar to the definition used by Hopster et al. (1998). From the definition it is clear that CCS is a measure of variation. This works so that if a cow changes box every time the CCS becomes zero (0.0) and if she keeps using the same box all the time the CCS becomes 1.0. If she makes completely random choices, then CCS becomes 0.5. The segment mean use of a specific milking box was obtained by recoding boxes as $\mathrm{A}=0.0$ and $\mathrm{B}=1.0$ and calculating mean values of that variable (Mean_box_ID). Thereby, a value Mean_box_ID at 0.5 indicates equal use of the 2 boxes. An illustration of the CCS calculation is given in Figure 1 for an example cow during 2 lactations. This cow gradually develops consistency during her first lactation by increasingly choosing milking box A. This continues into lactation 2 when it gets even more pronounced, and her CCS comes close to 1.0 .

Distributions of variables were scrutinized using QQ-plots. For the CCS and the other milking traits, approximate normal distributions of residuals were generally obtained. Data were allocated to 2 tables, one containing only first-parity records and another containing data from 1 lactation for each cow, chosen so that the lactation providing most data was preferred. If 2 lactations contained an equal number of useful seg- ments, the one with lowest parity number was chosen. This step of data reduction was taken to avoid having both between and within parity animal effects in the model.

Data were first analyzed one trait at a time using a linear mixed model, and then the model was extended to include 2 or more traits at a time and their possible co-variances. In general the form
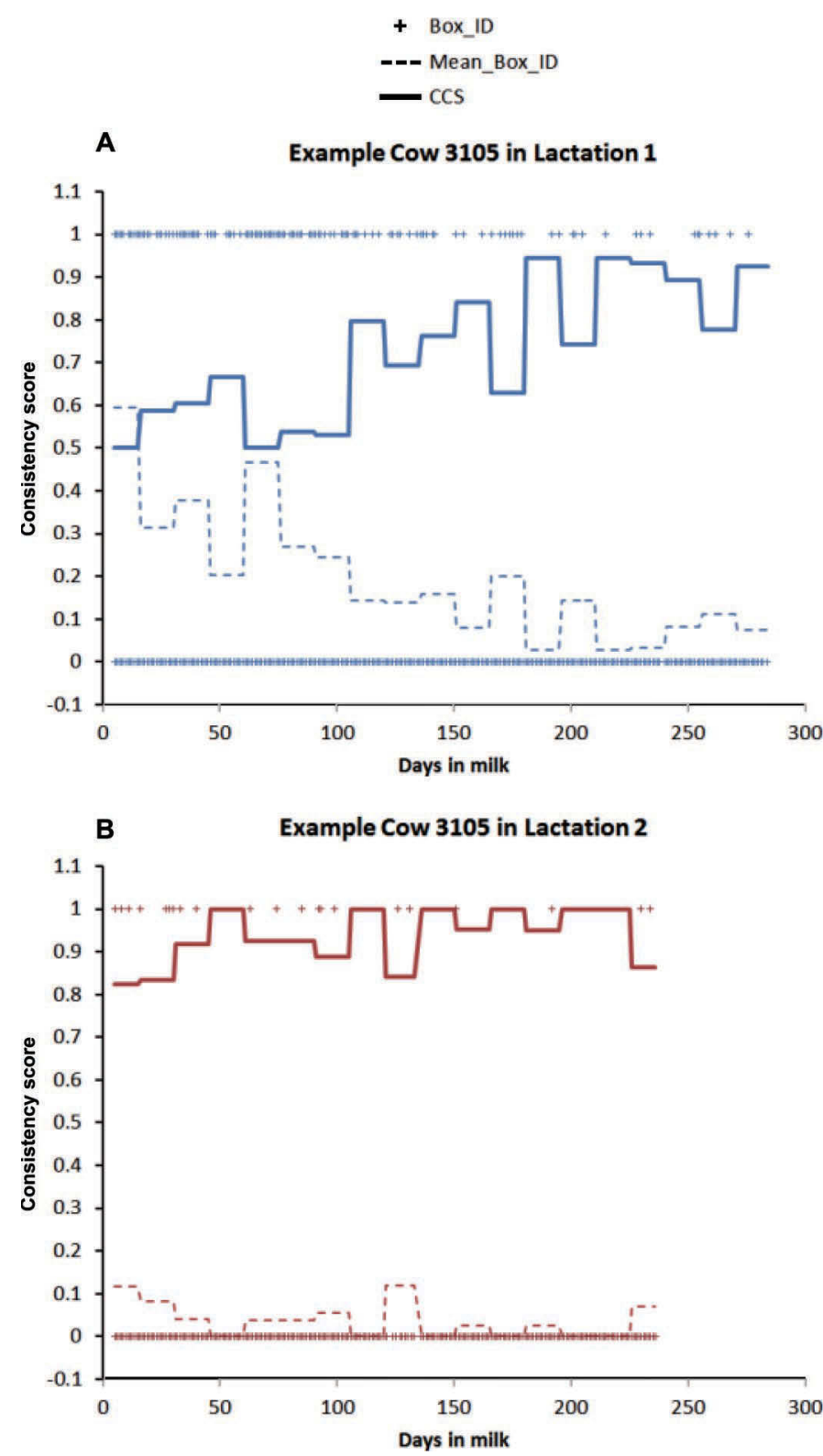

Figure 1. The patterns of milking box choice (Box_ID) for one particular example cow during her first 2 lactations, and the calculated choice consistency scores for 15 -d segments (CCS). The average milking box choice per segment is shown with broken lines (Mean_Box_ ID). Color version available online. 


$$
\mathbf{y}=\mathbf{X} \mathbf{b}+\mathbf{Z}_{1} \mathbf{u}_{1}+\mathbf{Z}_{2} \mathbf{u}_{2}+\mathbf{I} e
$$

was used, where $\mathbf{y}$ is a vector of observed traits; $\mathbf{X}$ is the design matrix for fixed effects and $\mathbf{b}$ the fixed effect solutions. The additive genetic effects are in vector $\mathbf{u}_{1}$ $\left[N\left(0, \sigma_{a}^{2}\right)\right]$, with additive genetic variance $\sigma_{a}^{2}$; permanent animal effect within lactations in $\mathbf{u}_{2}\left[N\left(0, \sigma_{p e}^{2}\right)\right]$, having permanent environmental variance $\sigma_{p e}^{2}$ and incidence matrices $\mathbf{Z}_{1}$ and $\mathbf{Z}_{2}$, with $\mathbf{Z}_{1}$ being the incidence matrix relating phenotypes to genetic effects and $\mathbf{Z}_{2}$ relating phenotypes to permanent environmental effects. Random residuals were in $e$, with incidence matrix $\mathbf{I}$. This model was also used in reduced versions, first to include only first parity data, and in versions focusing on individual level variance; thus, including the genetic effect $\mathbf{u}_{\mathbf{1}}$ into the permanent environmental animal effect $\mathbf{u}_{\mathbf{2}}$. The fixed effects in the model were, herd (5 levels), parity group (first or older), parity number (1 or 4 levels, because cows older than parity 4 were few and therefore omitted), segment, segment $\times$ parity group, and seasonal effects as herd-year-seasons. Fixed effects were initially tested using a linear mixed model where the genetic variance was not separated from the permanent animal effects, using the MIXED procedure of SAS (SAS 9.3, SAS Institute Inc., Cary, NC) analyzing one trait at a time.

Estimates of (co)variance components were used to calculate genetic parameters (heritability and genetic correlation), and permanent environmental covariance components were used for calculation of repeatability coefficients and individual level correlations between traits as well as phenotypic correlations. Estimates of covariance components were obtained using AI-REML in the DMU software package (DMU version 6 Release 4.7; Madsen and Jensen, 2008).

The average CCS was 0.63 in first parity, and similarly was 0.62 in multiple parity cows with a standard deviation of 0.15 . The CCS was slightly higher at start than in late and mid lactation sections (Table 1). The milking frequency was highest in the early lactation section and differences between early and late sections were largest in multiple-parity cows (Table 1). The CCS was rather similar across the 5 herds and 4 parities (Figure 2). The highest daily yields (MY24) followed the same changes as milking frequency through lactation and between parities, and similar developments were found for total box time per day. The average use of the 2 available milking units was similar for all subgroups in all 5 herds, indicating even use (not shown). The distribution of choice consistency showed that $3.5 \%$ of the cow segments were completely consistent $(\mathrm{CCS}=1.0)$. The remaining segments showed varying degrees of consistency, mostly between 0.25 and 1.00, approximately following a somewhat skewed normal distribution. No cows were completely inconsistent in their choices.

Automatically milked cows are often in herds with access to 2 or more milking boxes and, thus, must make a choice before each milking as to which box to access. This study has shown that some cows are highly consistent in choosing a specific milking box at the expense

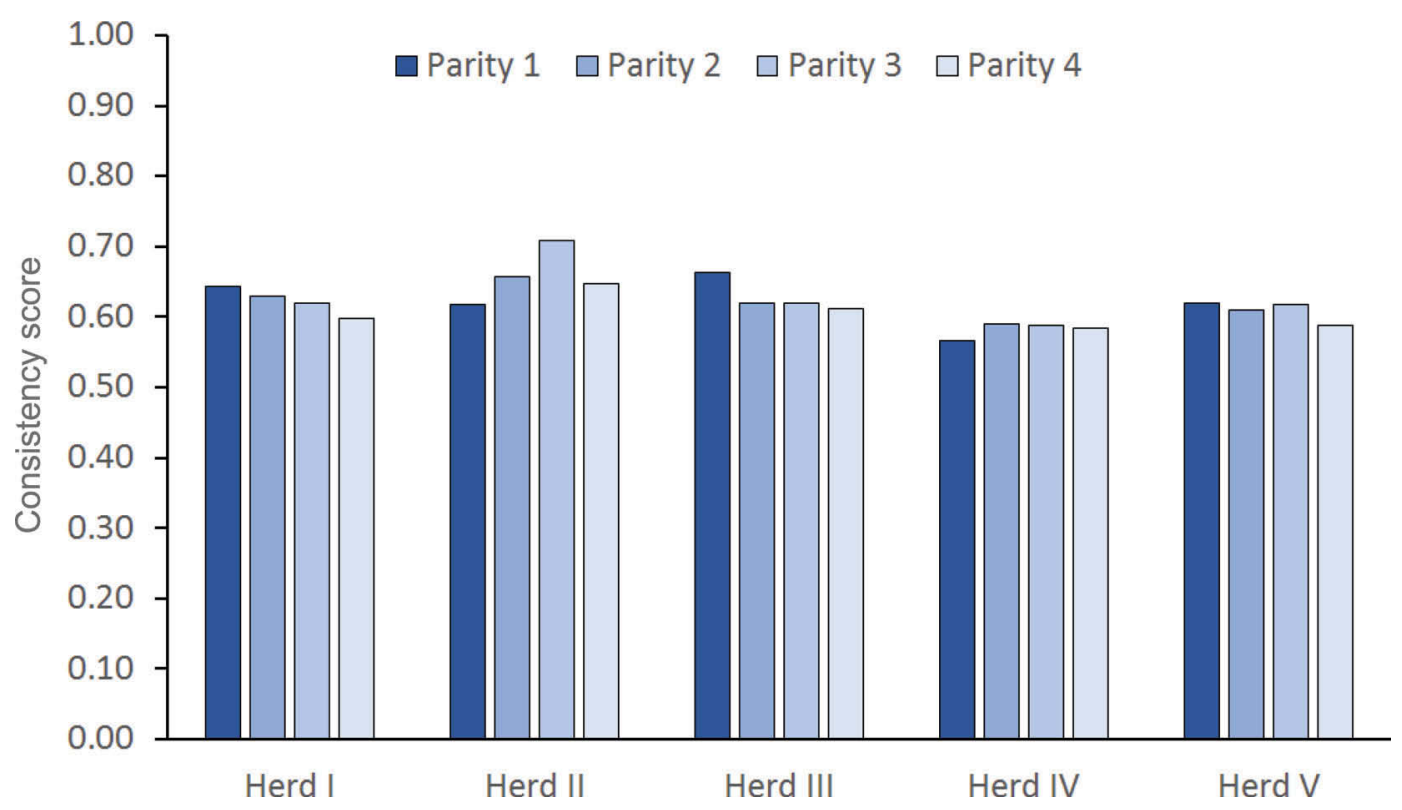

Figure 2. The mean consistency score per cow-segment for 4 parities within 5 herds. Color version available online. 
of waiting for it to be available; other cows seem to be flexible in their choice, perhaps just going for the first one open. From the data available for this study, no clues were available as to why one milking box was chosen before another by any specific cow. On the contrary, the average use of the 2 boxes in the studied herds was so similar that no overall preferences were detectable. This is in agreement with findings of strong side preferences in milking parlors and side preference being consistent over longer periods (Hopster et al., 1998).

Choice consistency was found to be a repeatable trait in both young and all cows. Although a lower repeatability was found in the start of first lactation, estimates of intermediate repeatability were found for all other lactation sections and of similar size in young and all cows (Table 2). The estimates of heritability were generally low, between 0.02 and 0.14 , and these estimates were not different from zero given calculated standard errors. For milk yield, estimates of heritability were lowest at start of lactation and then increased in mid and late lactation. Similarly, milking frequency showed intermediate heritability throughout lactation (Table 2). Time spent in the milking box was found to be highly heritable throughout all lactation stages and age classes.

The degree of consistency was moderately repeatable within lactation but had rather low heritability, indicating that individual effects are not predominantly caused by genetic background. If not caused by the genetic background another explanation can be a learned behavior (Grandin et al., 1994), which can persist over long stretches of time. Unfortunately, only interview information from herd managers was available and not data from the studied commercial herds explaining how heifers were trained to use milking boxes; thus, it can only be speculated that the training sessions before and in early lactation can work as a learning period. In behavior experiments, choice consistency has been used to assess if a learning process has been successful (e.g., Rioja-Lang et al., 2012). Another plausible explanation is that some cows had a bad (unrecorded) experience in one of the milking boxes and, therefore, try to avoid that in the future. Further explanations could include the hierarchy of cows, where high-ranking cows get access to their preferred box and lower ranking cows need to be more flexible. These explanations would all require further studies and data with more details than were available in the commercial herds.

This study has shown that some cows are highly consistent in their choice of milking box, and that being consistent is itself a consistent (repeatable) trait. However, genetic variation seems to play a minor role in determining how consistent cows are in this situation, as heritability estimates were generally low. The degree of consistency varies widely among cows, but shows no correlation to daily milk yield and time per day occupying the milking box.

This study has also shown that consistency has a low heritability. The performance traits analyzed in our study showed heritability estimates within the range expected from previous studies in commercial AMS herds using a test-day approach (e.g., Løvendahl and Chagunda, 2011). This indicates that the estimated heritability for CCS is low. By using segmentation of the lactation and analyzing it in sections it was shown that the heritability estimates were low all through the course of lactation and also that estimates were low both in first parity and when based on multiple-parity information.

Choice consistency scores were not significantly correlated with daily milk yield or box time at any age or at any lactation section at the individual or the phenotypic level. However, cows with high consistency score had lower milking frequency, as indicated by the weak

Table 2. Genetic parameter estimates for choice consistency score and concurrently recorded production traits, during 4 stages of lactation (DIM section), in primiparous and multiparous Holstein cows ${ }^{1}$

\begin{tabular}{|c|c|c|c|c|c|}
\hline \multirow[b]{2}{*}{ Item } & \multicolumn{2}{|c|}{$\begin{array}{l}\text { Choice consistency } \\
\text { score }\end{array}$} & \multirow{2}{*}{$\frac{\text { Milk yield }}{h^{2} \pm S E}$} & \multirow{2}{*}{$\frac{\text { Box time }}{h^{2} \pm \mathrm{SE}}$} & \multirow{2}{*}{$\frac{\text { Milking frequency }}{\mathrm{h}^{2} \pm \mathrm{SE}}$} \\
\hline & $t \pm \mathrm{SE}$ & $\mathrm{h}^{2} \pm \mathrm{SE}$ & & & \\
\hline \multicolumn{6}{|l|}{ Primiparous } \\
\hline 5-50 DIM & $0.27 \pm 0.04$ & $0.04 \pm 0.06$ & $0.09 \pm 0.10$ & $0.51 \pm 0.14$ & $0.21 \pm 0.10$ \\
\hline 51-110 DIM & $0.52 \pm 0.03$ & $0.11 \pm 0.08$ & $0.14 \pm 0.11$ & $0.41 \pm 0.15$ & $0.18 \pm 0.12$ \\
\hline 111-215 DIM & $0.50 \pm 0.02$ & $0.14 \pm 0.08$ & $0.28 \pm 0.12$ & $0.45 \pm 0.13$ & $0.15 \pm 0.10$ \\
\hline \multicolumn{6}{|l|}{ Multiparous } \\
\hline 5-50 DIM & $0.53 \pm 0.02$ & $0.05 \pm 0.04$ & $0.13 \pm 0.07$ & $0.48 \pm 0.09$ & $0.21 \pm 0.07$ \\
\hline 51-110 DIM & $0.56 \pm 0.02$ & $0.07 \pm 0.05$ & $0.24 \pm 0.08$ & $0.47 \pm 0.09$ & $0.30 \pm 0.08$ \\
\hline 111-215 DIM & $0.53 \pm 0.02$ & $0.07 \pm 0.04$ & $0.30 \pm 0.08$ & $0.49 \pm 0.09$ & $0.29 \pm 0.07$ \\
\hline 216-305 DIM & $0.51 \pm 0.02$ & $0.02 \pm 0.03$ & $0.37 \pm 0.09$ & $0.38 \pm 0.09$ & $0.27 \pm 0.08$ \\
\hline
\end{tabular}

${ }^{1}$ Results are repeatability $(t)$ and heritability $\left(\mathrm{h}^{2}\right)$ given with approximate SE. 
Table 3. Correlations between choice consistency score (CCS) and production traits during 4 stages of lactation (DIM section), in primiparous and multiparous Holstein cows ${ }^{1}$

\begin{tabular}{|c|c|c|c|c|c|c|}
\hline \multirow[b]{2}{*}{ Item } & \multicolumn{2}{|c|}{ Milk yield } & \multicolumn{2}{|c|}{ Box time } & \multicolumn{2}{|c|}{ Milking frequency } \\
\hline & $\mathrm{r}_{\mathrm{i}} \pm \mathrm{SE}$ & $r_{p}$ & $\mathrm{r}_{\mathrm{i}} \pm \mathrm{SE}$ & $r_{p}$ & $\mathrm{r}_{\mathrm{i}} \pm \mathrm{SE}$ & $\mathrm{r}_{\mathrm{p}}$ \\
\hline 5-50 DIM & $-0.03 \pm 0.07$ & 0.00 & $0.11 \pm 0.07$ & 0.06 & $-0.21 \pm 0.08$ & -0.07 \\
\hline 51-110 DIM & $0.00 \pm 0.06$ & 0.00 & $0.00 \pm 0.06$ & -0.01 & $-0.14 \pm 0.06$ & -0.10 \\
\hline 111-215 DIM & $0.00 \pm 0.05$ & 0.00 & $0.00 \pm 0.05$ & 0.00 & $-0.15 \pm 0.05$ & -0.11 \\
\hline 216-305 DIM & $0.10 \pm 0.05$ & 0.02 & $-0.03 \pm 0.05$ & -0.03 & $-0.09 \pm 0.05$ & -0.07 \\
\hline 51-110 DIM & $-0.02 \pm 0.04$ & -0.01 & $-0.02 \pm 0.04$ & -0.03 & $-0.16 \pm 0.04$ & -0.12 \\
\hline 111-215 DIM & $-0.02 \pm 0.04$ & -0.01 & $0.01 \pm 0.04$ & 0.00 & $-0.11 \pm 0.04$ & -0.08 \\
\hline 216-305 DIM & $0.02 \pm 0.04$ & 0.01 & $0.03 \pm 0.04$ & 0.00 & $-0.08 \pm 0.04$ & -0.07 \\
\hline
\end{tabular}

${ }^{1}$ Results are shown as individual level $\left(\mathrm{r}_{\mathrm{i}}\right)$ and phenotypic correlations $\left(\mathrm{r}_{\mathrm{p}}\right)$

negative correlations at both individual and phenotypic level (Table 3). This indicates that cows with a preference for a specific milking box spend time waiting for that box to become available, but the extra waiting time is not affecting daily milk yield or the total time per day occupying the milking box.

Milk yield per day and milking frequency are in other studies shown to be positively correlated (e.g., Løvendahl and Chagunda, 2011), but in this case the effect of lower milking frequency in highly consistent cows seems to be fully compensated so that the milk yield per day is unaffected by consistency score. This is less likely to be a genetic selection effect, because AMS has only been around for about $15 \mathrm{yr}$ and only recently the coverage of Danish cows exceeded 25\% (Løvendahl and Chagunda, 2011). In previous studies it was reported that cows that were forced to be milked in the nonpreferred side of a parlor had reduced yield (Hopster et al., 1998). A similar effect could not be tested here because data were collected entirely from milkings that were categorized as automated and completed, ensuring that cows were in a free-choice situation.

The results of our study show large variation exists within a herd as to how consistent cows are in their choices. However, no clear explanation exists why some cows develop high choice consistency whereas others do not. Some studies have shown that cows' responses to a range of stimuli are somehow correlated, indicating a consistency across situations (Müller and Schrader, 2005; Gibbons et al., 2009); but the continuous and approximately normal distribution of consistency scores show that there are no distinct choice strategies to which each cow can be assigned, even for shorter time periods. Rather, it seems that a mixture of strategies exists within each AMS herd, and that no single one of these strategies is superior to others in terms of production traits.
The consequence of having cows that are highly consistent in their choice should be considered in the designs of barn layout and instrumentation as well as in herd management. For example, waiting areas need to be large enough so that cows can wait for their favorite milking box to become available. Also, if one wants to take milk samples from all cows in the herd, samplers must be installed in all available milking boxes to obtain complete coverage of all cows. Similarly, if expensive equipment is to be installed, all available units must be serviced to ensure full coverage.

The present study used CCS to study animal choice in cows in a simple situation; this could extend to other situations with more choices or with different preferences to the possible choices. It would also be interesting to apply the different situations and compare consistency within and between different situations (Müller and Schrader, 2005; Gibbons et al., 2009). A range of other models could also be applied to data of this kind, including time series models, and models using the binomial nature of choice events. However, for estimation of genetic parameters, linear mixed models were chosen because the traits were approximately normally distributed in the way they were calculated.

In conclusion, a strong individuality in choice consistency was found for automatically milked cows in their preference for 1 of 2 possible milking boxes in 5 commercial herds. Although quantitative genetic variation affected choice consistency, this gave only a low heritability; however, choice consistency was almost uncorrelated with production traits, except for milking frequency.

\section{ACKNOWLEDGMENTS}

We are grateful to the owners of the 5 commercial herds who provided us with data for this study. The 
study received financial support from various funds, including Danish Strategic Research Council (Copenhagen, Denmark), Danish Cattle Organization (Skejby, Denmark), and the Danish Ministry of Food Fisheries and Agriculture (Copenhagen, Denmark).

\section{REFERENCES}

Gibbons, J. M., A. B. Lawrence, and M. J. Haskell. 2009. Consistency of aggressive feeding behaviour in dairy cows. Appl. Anim. Behav. Sci. 121:1-7.

Grandin, T., K. G. Odde, D. N. Schutz, and L. M. Behrns. 1994. The reluctance of cattle to change a learned choice may confound preference tests. Appl. Anim. Behav. Sci. 39:21-28.

Grasso, F., G. De Rosa, F. Napolitano, A. Di Franca, and A. Bordi. 2007. Entrance order and side preference of dairy cows in the milking parlour. Ital. J. Anim. Sci. 6:187-194.

Hopster, H., J. T. N. van der Werf, and H. Blokhuis. 1998. Side preferences of dairy cows in the milking parlour and its effects on behaviour and heart rate during milking. Appl. Anim. Behav. Sci. $55: 213-229$.

Løvendahl, P., and M. G. G. Chagunda. 2011. Covariance among milking frequency, milk yield, and milk composition from automatically milked cows. J. Dairy Sci. 94:5381-5392.
Løvendahl, P., L. Damgaard, B. L. Nielsen, K. Thodberg, G. Su, and L. Rydhmer. 2005. Aggressive behaviour of sows at mixing and maternal behaviour are heritable and genetically correlated traits. Livest. Prod. Sci. 93:73-85.

Løvendahl, P., J. Lassen, and M. G. G. Chagunda. 2012. Genetic variation in milking efficiency: A novel trait for milkability in automatic milking systems. Page 172 in EAAP Book of Abstracts, Vol. 18. Wageningen Academic Publishers, Wageningen, the Netherlands.

Løvendahl, P., and L. Munksgaard. 2016. An investigation into genetic and phenotypic variation in time budgets of dairy cows. J. Dairy Sci. 99:408-417.

Madsen, P., and J. Jensen. 2008. A user's guide to DMU: A package for analysing multivariate mixed models. Ver. 6, rel. 4.7. Aarhus University, Foulum, Denmark.

Müller, R., and L. Schrader. 2005. Individual consistency of dairy cows' activity in their home pen. J. Dairy Sci. 88:171-175.

Paranhos da Costa, M. J. R., and D. M. Broom. 2001. Consistency of side choice in the milking parlour by Holstein-Friesian cows and its relationship with their reactivity and milk yield. Appl. Anim. Behav. Sci. 70:177-186.

Rioja-Lang, F. C., D. J. Roberts, S. D. Healey, A. B. Lawrence, and M. J. Haskell. 2012. Dairy cow feeding space requirements assessed in a Y-maze choice test. J. Dairy Sci. 95:3954-3960. 